\title{
Effective properties and eddy current losses of soft magnetic composites
}

Cite as: J. Appl. Phys. 129, 015103 (2021); https://doi.org/10.1063/5.0031226

Submitted: 28 September 2020 . Accepted: 13 December 2020. Published Online: 05 January 2021

Romain Corcolle, (Diaotao Ren, and (iD) Laurent Daniel
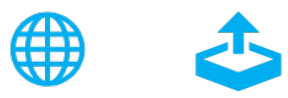

\section{ARTICLES YOU MAY BE INTERESTED IN}

Microwave Hall effect measurement for materials in the skin depth region

Journal of Applied Physics 129, 015102 (2021); https://doi.org/10.1063/5.0033777

Transformation-induced plasticity in omega titanium

Journal of Applied Physics 129, 015105 (2021); https://doi.org/10.1063/5.0035465

Magnetic textures in hemispherical thin film caps with in-plane exchange bias

Journal of Applied Physics 129, 015305 (2021); https://doi.org/10.1063/5.0033772

\section{Challenge us.}

What are your needs for periodic signal detection?

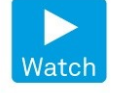

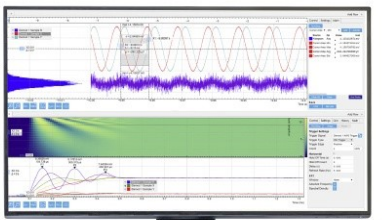

-
Zurich

Instruments 


\title{
Effective properties and eddy current losses of soft magnetic composites
}

\author{
Cite as: J. Appl. Phys. 129, 015103 (2021); doi: 10.1063/5.0031226 \\ Submitted: 28 September 2020 . Accepted: 13 December 2020 . \\ Published Online: 5 January 2021

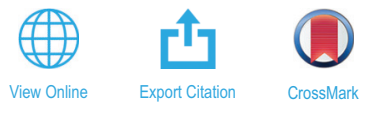

Romain Corcolle, ${ }^{1, a)}$ (I) Xiaotao Ren, ${ }^{2}$ (D) and Laurent Daniel ${ }^{3,4}$ (D)

\begin{abstract}
AFFILIATIONS
'Division of Engineering and Computer Science, NYU Shanghai, 1555 Century Avenue, Shanghai 200122, People's Republic of China

${ }^{2}$ Integrated Actuators Laboratory (LAI), École Polytechnique Fédérale de Lausanne (EPFL), Rue de la Maladière 71B, Neuchâtel 2000, Switzerland

${ }^{3}$ Laboratoire de Génie Electrique et Electronique de Paris, Université Paris-Saclay, CentraleSupélec, CNRS, 91192 Gif-sur-Yvette, France

${ }^{4}$ Laboratoire de Génie Electrique et Electronique de Paris, Sorbonne Université, CNRS, 75252 Paris, France
\end{abstract}

a) Author to whom correspondence should be addressed: rc173@nyu.edu

\begin{abstract}
This paper presents a semi-analytical homogenization model for Soft Magnetic Composites (SMCs), providing the effective magnetic behavior and the level of Eddy Current (EC) losses. Both linear and nonlinear magnetic behavior are considered. A magnetic circuit made of SMC is then modeled with a Finite Element Model (FEM). The size of heterogeneities of SMC being much smaller than the device size, the proposed approach relieves the burden of a very fine mesh in the FEM, by using the effective properties of an equivalent homogeneous material. The approach is validated by comparing the results on the homogenized magnetic circuit with the ones obtained from a computationally heavy FEM describing the heterogeneities with a very fine mesh. The results show that the homogenized model provides a very accurate description of the magnetic behavior and EC losses of SMC for both linear and nonlinear cases.
\end{abstract}

Published under license by AIP Publishing. https://doi.org/10.1063/5.0031226

\section{INTRODUCTION}

Soft Magnetic Composites (SMCs) are a new type of material created by powder metallurgy technology or $3 \mathrm{D}$ printing. ${ }^{1}$ They consist in a collection of ferromagnetic particles embedded in a dielectric insulating polymer matrix. Ferromagnetic materials ensure a relatively high permeability of the composite while the dielectric matrix confines the Eddy Current (EC) within each particle, thus greatly reducing the EC losses of the composite. Contacts between particles may happen, thus increasing the permeability of the composite ${ }^{2}$ but also increasing EC losses. Continuous research on SMC has shown a wide range of applications. ${ }^{3,4}$ To design electric devices using SMC, the effective magnetic behavior and the losses are the two dominant points to take into consideration.

Losses in magnetic materials can be classically separated into three components: ${ }^{5-7}$ static hysteresis, EC losses (also named classical losses), and excess losses. On one hand, static hysteresis and excess losses in SMC are local effects related to domain-wall movements in the ferromagnetic particles. The typical scale for the description of static and excess losses is the micrometer (magnetic domain scale).
Extensions of the standard statistical theory of losses, based on loss separation, to the case of SMC have been proposed. ${ }^{8}$ However, if the standard approaches can be applied to static and excess components of losses, EC losses on the other hand are strongly related to the microstructure of the composite. They mainly depend on the particle shapes and sizes, and also on the contacts between particles. Models for estimating the losses in SMC already exist and can be categorized into two main streams: numerical approaches vs analytical approaches.

Numerical models, usually based on Finite Element Methods (FEMs), are very powerful approaches, which can fully describe the dependence of the macroscopic magnetic behavior ${ }^{2,9}$ and the EC losses $^{10-13}$ upon the microstructure. Such models can consider the nonlinear magnetic behavior of the ferromagnetic particles, but these approaches are computationally expensive. For example, a statistical study on the microstructure parameters (shape, size, and arrangement of inclusions) requires to run the model for each realization of the microstructure, which would lead to a very expensive computation. 
Analytical or semi-analytical models are computationally light approaches, which can be easily implemented in the studies of electromagnetic devices made of SMC. However, their range of validity is sometimes considered limited compared to numerical approaches. This is probably due to the fact that most applications of analytical models deal with linear magnetic behavior, periodic microstructures, perfect insulation between particles, or low frequency range. However, as will be shown in this paper, the range of validity of analytical and semi-analytical models can be extended to a broader set of assumptions.

Many analytical models for SMC are based on the definition of a complex permeability in the harmonic regime. Effective complex electromagnetic properties are a useful tool to model the behavior of composites with linear properties. ${ }^{14}$ Effective complex permeability, permittivity, or conductivity can be used to study the electromagnetic behavior of composites in various applications. ${ }^{15-17}$ Complex permeability deals easily with high frequency magnetic effects for magnetic circuit applications ${ }^{18,19}$ where the imaginary part directly reflects the power dissipation. ${ }^{20-22}$ Complex permeability models have also been applied to homogenize the multi-turn coil of magnetic circuits ${ }^{23}$ and can consider the skin effect. A similar approach has been applied to the periodic SMC with circular or spherical inclusions at low frequency ${ }^{24}$ but limited so far to relatively low ferromagnetic particle concentrations. Another category of analytical models can also deal with the nonlinear magnetization of SMC. ${ }^{25}$ But no analytical model provides a description of EC losses in the case of nonlinear magnetic behavior.

In this paper, the equivalent complex permeability approach is first used for high concentration SMC with a periodic microstructure and linear magnetic behavior. A more advanced model, still based on analytical formulas, is then developed for considering nonlinear magnetization of iron inclusions. This model also enables the estimation of EC losses. Last, the developed homogenized model of SMC is implemented in FEM for the study of a magnetic circuit in order to evaluate their relevance to engineering applications.

\section{HOMOGENIZATION}

The study case for this paper is a magnetic circuit as depicted in Fig. 1.

It is made of high concentration periodic SMC with square iron inclusions. The copper coils are wound to provide the magnetic field input to the magnetic circuit.

In Secs. II B and II C, the homogenization models are presented. First, the magnetic behavior of the periodic cell is homogenized with a classical mixing rule. In a second step, the EC loss density of the periodic cell is estimated.

\section{A. Microstructure}

SMCs exhibit 3D microstructures, but this study will only consider a 2D magnetic circuit made of 2D SMC (with infinitely long inclusions along the third direction). The proposed homogenization approach still applies to $3 \mathrm{D}$ microstructures, but the FEM study of a 3D magnetic circuit made of 3D SMC would require to solve a very heavy numerical system. The goal of this study is to evaluate the performance of the homogenization model, which can be achieved with $2 \mathrm{D}$ structures.

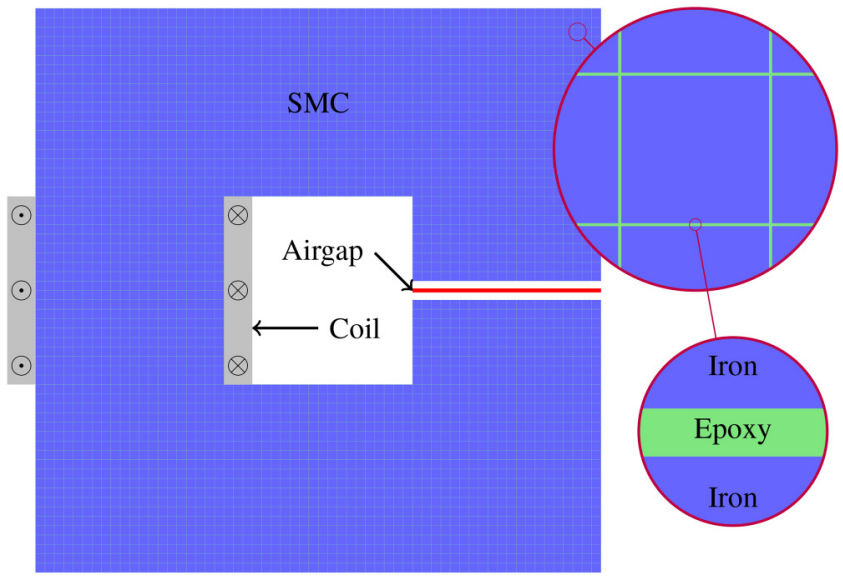

FIG. 1. Sketch of a magnetic circuit made of SMC with square iron inclusions.

The 2D SMC problem can be separated into two different cases depending on the direction of the magnetic field excitation. The first case considers an applied magnetic field perpendicular to the $2 \mathrm{D}$ domain leading to a uniform magnetic field. Corresponding analytical or semi-analytical models have already been proposed with a similar approach to calculate EC losses for laminated steel ${ }^{26}$ and for SMC. ${ }^{27}$ This case is relatively easy to homogenize with a Wiener mixing rule. ${ }^{28}$ Nonlinear magnetic behavior can be handled with a simple secant linearization scheme.

The second case considers the magnetic field in the $2 \mathrm{D}$ plane. In that case, the magnetic field is generally nonuniform in the different phases. Numerical methods can usually provide an accurate description of the magnetic field but analytical mixing rules for linear behavior (based on mean field approaches) also exist. For nonlinear behavior, homogenization is usually more complicated because the distribution of the magnetic field needs to be accurately determined but accurate estimates for the macroscopic magnetic behavior can still be built. ${ }^{25,29}$ For example, semi-analytical models can be based on various secant or tangent linearization schemes.

In our study, the microstructure is well defined as a periodic cell made of a square inclusion of iron (permeability $\mu_{2}$, or nonlinear magnetization curve, and conductivity $\sigma_{2}$ ) insulated by a thin layer of epoxy (permeability $\mu_{1}$, null conductivity). Figure 2 indicates the geometric properties of the unit cell, representing a filling factor of iron inclusions $\xi_{2}$ of $96.04 \%$.

\section{B. Magnetic behavior}

The homogenized magnetic behavior can be described by the effective permeability $\widetilde{\mu}$, which links the macroscopic magnetic field $\overline{\mathbf{H}}$ to the macroscopic magnetic induction $\overline{\mathbf{B}}$ over the unit cell,

$$
\overline{\mathbf{B}}=\langle\mathbf{B}(\mathbf{x})\rangle=\langle\mu(\mathbf{x}) \cdot \mathbf{H}(\mathbf{x})\rangle=\widetilde{\mu} \cdot\langle\mathbf{H}(\mathbf{x})\rangle=\widetilde{\mu} \cdot \overline{\mathbf{H}},
$$

where $\langle\cdot\rangle$ is a volume average operator over the unit cell, $\mathbf{x}$ is the position vector on the unit cell, $\mu(\mathbf{x})$ is the local permeability, 


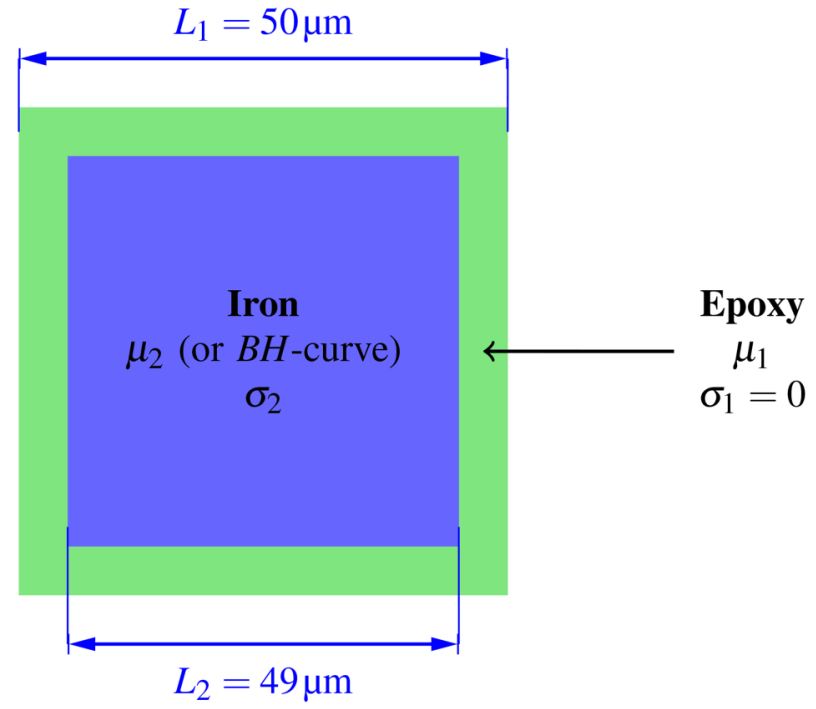

FIG. 2. Geometric parameters of the periodic cell of SMC with square iron inclusions (the scale is not respected).

$\mathbf{H}(\mathbf{x})$ is the local magnetic field, and $\mathbf{B}(\mathbf{x})$ is the local magnetic induction.

In the case of linear magnetic behavior, $\widetilde{\mu}$ is constant. In the case of nonlinear magnetic behavior, $\widetilde{\mu}$ depends on the magnitude of the magnetic loading.

\section{Linear behavior}

For a small magnitude of loadings, the iron magnetic behavior can be modeled with a linear constitutive law. In such a case, the classical Maxwell Garnett (MG) estimate $\widetilde{\mu}_{M G}$ is an accurate model for the effective permeability, ${ }^{30}$

$$
\widetilde{\mu}_{M G}=\frac{\left(\mu_{2}+\mu_{1}\right)+\left(\mu_{2}-\mu_{1}\right) \xi_{2}}{\left(\mu_{2}+\mu_{1}\right)-\left(\mu_{2}-\mu_{1}\right) \xi_{2}} \mu_{1} .
$$

This estimate was originally developed for the case of dilute ellipsoidal inclusions, but it is also an accurate estimate in this study since the magnetic field is quasi-uniform in the inclusions because of the high volume fraction $\left(\xi_{2}>95 \%\right)$ of inclusions.

As long as the frequency is low enough, the magnetic field induced by EC can be neglected and the assumption of a uniform magnetic field in the inclusions is valid. For high frequencies, this assumption is not valid anymore.

The limitation of the model regarding the frequency will be discussed in Sec. III.

\section{Nonlinear behavior}

For higher magnitudes of magnetic loading, iron magnetic behavior may reach saturation (see Fig. 3).

Similarly to the linear case, the assumption of a uniform magnetic field in the inclusions is still valid for nonlinear magnetic

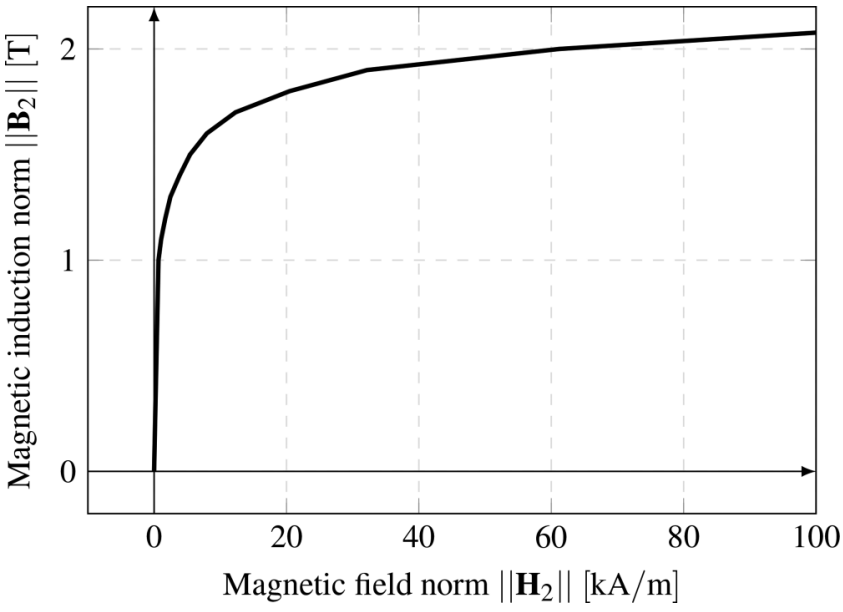

FIG. 3. Magnetization curve of iron.

behavior, because of the very high filling factor of inclusions. Therefore, the whole inclusion behavior can be linearized into a unique secant permeability value $\mu_{2}^{s}$, which depends on the magnetic field in the inclusion $\mathbf{H}_{2}$. MG estimate will then give an accurate effective permeability value depending on the macroscopic loading (the macroscopic magnetic field $\overline{\mathbf{H}}$ ).

This problem can be solved iteratively, thanks to the localization $^{32}$ of the magnetic field $\mathbf{H}_{2}$ in the inclusions ${ }^{33}$ (see Fig. 4).

\section{EC losses}

The EC loss density $U$ is defined as the average Joule losses dissipated on the unit cell during a wave period,

$$
U=\left\langle\int_{0}^{T} \mathbf{J}(\mathbf{x}, t) \cdot \mathbf{E}(\mathbf{x}, t) d t\right\rangle
$$

where $T$ is the period of the loading (frequency $f$ ), $\mathbf{J}(\mathbf{x}, t)$ is the local current density, and $\mathbf{E}(\mathbf{x}, t)$ is the local electric field. The current density $\mathbf{J}(\mathbf{x}, t)$ relates to the electric field $\mathbf{E}(\mathbf{x}, t)$ through the local electrical conductivity $\sigma(\mathbf{x})$,

$$
\mathbf{J}(\mathbf{x}, t)=\sigma(\mathbf{x}) \cdot \mathbf{E}(\mathbf{x}, t)
$$

Since epoxy exhibits a very low conductivity (considered null in this study), the EC loss density for a unit cell can be written as

$$
U=\xi_{2} \sigma_{2}\left\langle\int_{0}^{T} \mathbf{E}(\mathbf{x}, t)^{2} d t\right\rangle_{2},
$$

with $\langle\cdot\rangle_{2}$ the volume average operator over the iron particle only.

\section{Linear behavior}

When the magnetic loading is harmonic and under the assumption of linear magnetic behavior, then the induced electric field is 


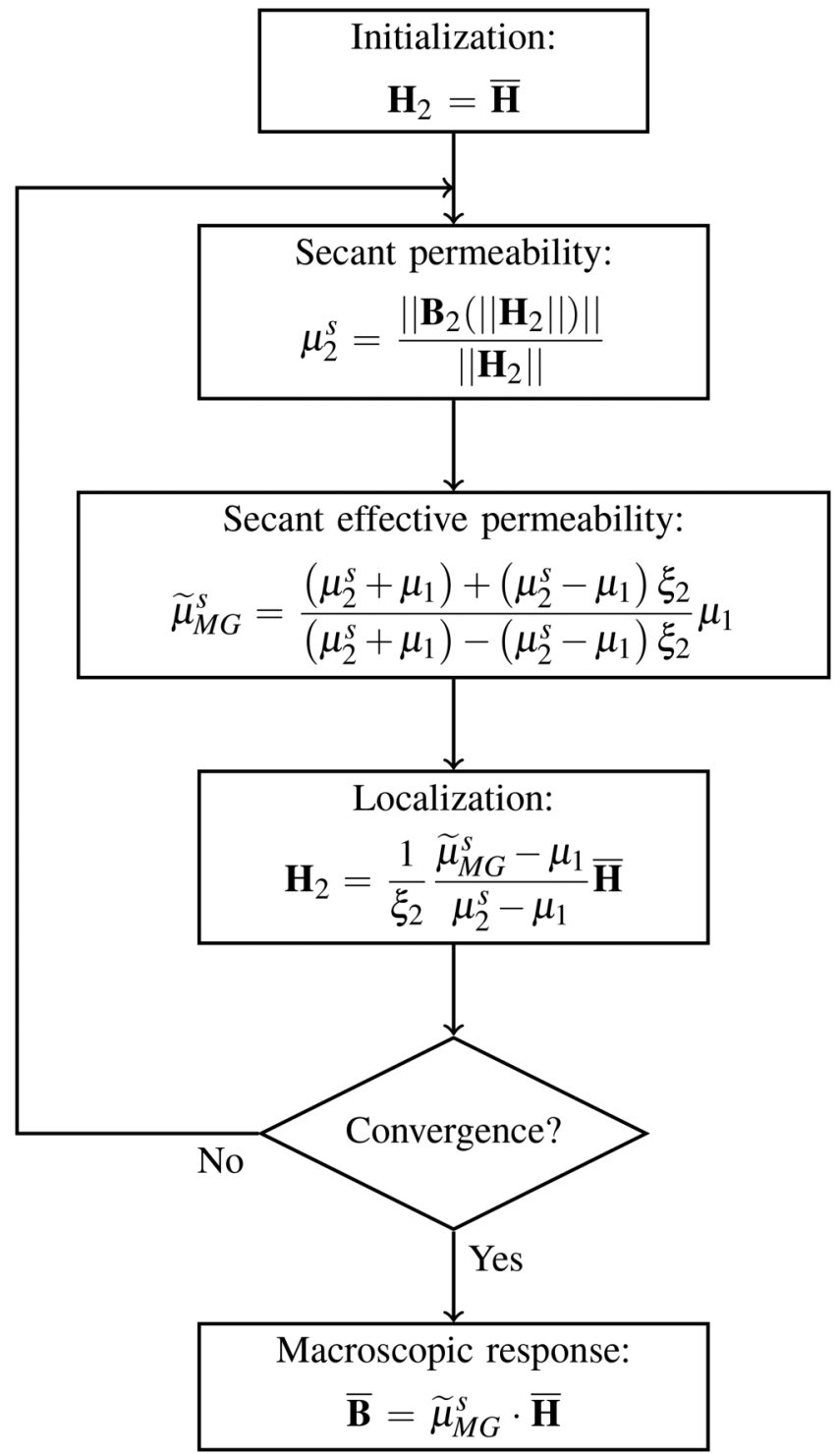

FIG. 4. Iterative scheme for the determination of the macroscopic nonlinear magnetic behavior.

also harmonic and the time integral in Eq. (5) can be modified as

$$
U=\frac{\xi_{2} \sigma_{2}}{2 f}\left\langle\underline{\mathbf{E}_{2}^{*}}(\mathbf{x}) \cdot \underline{\mathbf{E}_{2}}(\mathbf{x})\right\rangle_{2}
$$

where $\mathbf{E}_{2}(\mathbf{x})$ is the local complex magnitude of the electric field in the iron inclusion, and superscript ${ }^{*}$ indicates a complex conjugate operation.

Under the same assumption that the magnetic field $\mathbf{H}_{2}$ (with complex magnitude components $H_{2 x}$ and $H_{2 y}$ in the Cartesian coordinate system) in iron particles is uniform (assumption used for the determination of the effective permeability), the induced electric field $\mathbf{E}_{2}(\mathbf{x})$ in the iron square inclusion is equal to

$$
\underline{\mathbf{E}_{2}}(\mathbf{x})=-j 2 \pi f \mu_{2}\left(\underline{H_{2 x}} y-\underline{H_{2 y}} x\right) \vec{u}_{z},
$$

where $x$ and $y$ are the Cartesian coordinates (with origin at the center of the square inclusion), and $\overrightarrow{u_{z}}$ is the unit vector perpendicular to the $2 \mathrm{D}$ domain.

Replacing the electric field in the inclusion into Eq. (6), and averaging over the iron inclusion leads to

$$
U=\frac{\pi^{2}}{6} f \xi_{2} L_{2}^{2} \sigma_{2} \mu_{2}^{2} \underline{\mathbf{H}_{2}^{*}} \cdot \underline{\mathbf{H}_{2}} .
$$

This expression can be written as a function the macroscopic complex magnetic field magnitude $\overline{\mathbf{H}}$ by the same localization rule shown in Fig. 4 using the effective permeability $\widetilde{\mu}_{M G}$ from Eq. (2). Finally, the EC loss density is equal to

$$
U=\frac{2 \pi^{2}}{3} f \xi_{2} L_{2}^{2} \sigma_{2}\left(\frac{\mu_{1} \mu_{2}}{\left(\mu_{2}+\mu_{1}\right)-\left(\mu_{2}-\mu_{1}\right) \xi_{2}}\right)^{2} \overline{\mathbf{H}}^{*} \cdot \overline{\mathbf{H}} .
$$

Analytical homogenization models ${ }^{23}$ can also deal with a heterogeneous magnetic field distribution in iron particles due to skin effect at high frequencies, but such approaches are only valid for linear magnetic behavior. When nonlinear magnetic behavior is considered, the use of a numerical approach is unavoidable (for example, Cauer-Circuit elements obtained through a FEM ${ }^{12}$ ).

\section{Nonlinear behavior}

In the case of nonlinear magnetic behavior of iron, the induced electric field is not sinusoidal anymore under harmonic loading, meaning that Eq. (6) does not hold anymore.

Using again the assumption that the magnetic field $\mathbf{H}_{2}(t)$ [or magnetic induction $\mathbf{B}_{2}(t)$, with components $B_{2 x}(t)$ and $B_{2 y}(t)$ in the Cartesian coordinate system] is uniform in the iron inclusion, the electric field $\mathbf{E}(\mathbf{x}, t)$ in the inclusion is equal to

$$
\mathbf{E}(\mathbf{x}, t)=-\left(\frac{\partial B_{2 x}(t)}{\partial t} y-\frac{\partial B_{2 y}(t)}{\partial t} x\right) \vec{u}_{z} .
$$

Then, evaluating the volume average operation in Eq. (5) leads to

$$
U=\frac{\xi_{2} \sigma_{2} L_{2}^{2}}{12} \int_{0}^{T}\left(\frac{\partial \mathbf{B}_{2}(t)}{\partial t}\right)^{2} d t
$$

$\mathbf{B}_{2}(t)$ can be expressed as a function of the macroscopic induction $\overline{\mathbf{B}}(t)$ thanks to a localization rule similar to the one in Fig. $4,{ }^{32}$

$$
\mathbf{B}_{2}(t)=\frac{1}{\xi_{2}} \frac{1-\frac{\mu_{1}}{\widetilde{\mu}_{M G}^{s}}}{1-\frac{\mu_{1}}{\mu_{2}^{s}}} \overline{\mathbf{B}}(t)=\alpha \overline{\mathbf{B}}(t),
$$

with $\alpha$ a coefficient depending on $\overline{\mathbf{B}}(t)$. 
In the studied case in this paper, for a volume fraction of inclusion of $96.04 \%$ and with the magnetization curve shown in Fig. 3, $\alpha$ can be approximated to a constant value. Indeed, because in most cases $\mu_{2}^{s} \gg \mu_{1}$, one can write

$$
\alpha \approx \frac{2}{1+\xi_{2}}
$$

Finally, given the macroscopic magnetic induction $\overline{\mathbf{B}}(t)$ over a time period, the EC loss density for a unit cell can be retrieved with the following expression:

$$
U=\frac{\xi_{2} \sigma_{2} L_{2}^{2} \alpha^{2}}{12} \int_{0}^{T}\left(\frac{\partial \overline{\mathbf{B}}(t)}{\partial t}\right)^{2} d t .
$$

\section{RESULTS}

In order to validate the model, a single unit cell will be first modeled by FEM and the results will be compared to the homogenized formulas determined in Sec. II.

In a second time, a magnetic circuit made of SMC (similar to the one shown in Fig. 1) will be modeled by FEM with two approaches: one modeling the iron inclusions, and one using homogenized properties. The comparison of the results will evaluate the relevance of homogenized properties for the study of electromagnetic devices made of SMC.

\section{A. Parameters}

Iron behavior is characterized by an electrical conductivity $\sigma_{2}=1.12 \times 10^{7} \mathrm{~S} / \mathrm{m}$. The magnetic behavior is shown in Fig. 3 for the nonlinear behavior or by a constant permeability $\mu_{2}=1200 \mu_{0}$ ( $\mu_{0}$ is the vacuum permeability) for the linear models. Epoxy will be modeled by a null electrical conductivity and a permeability $\mu_{1}=\mu_{0}$. The relative dielectric permittivity is set to 1 for both materials.

\section{B. Unit cell}

A unit cell constituted of a single iron inclusion embedded in epoxy is modeled with FEM. When magnetic linear behavior is considered for iron inclusions, a harmonic study is used, leading to complex solutions. When nonlinear magnetic behavior is considered, a time-domain study is used.

\section{Magnetic field distribution}

The models developed in the previous section strongly rely on the assumption of a uniform magnetic field/induction in the iron inclusion.

In order to assess the validity of this assumption, the uniformity of the magnetic field $\mathbf{H}(\mathbf{x})$ in the iron particle is evaluated according to its variance $V_{2}$,

$$
V_{2}=\frac{\left\langle\underline{\mathbf{H}}^{*}(\mathbf{x}) \cdot \underline{\mathbf{H}}(\mathbf{x})\right\rangle_{2}-\left\langle\underline{\mathbf{H}}^{*}(\mathbf{x})\right\rangle_{2} \cdot\langle\underline{\mathbf{H}}(\mathbf{x})\rangle_{2}}{\langle\underline{\mathbf{H}}(\mathbf{x})\rangle_{2} \cdot\langle\underline{\mathbf{H}}(\mathbf{x})\rangle_{2}} .
$$

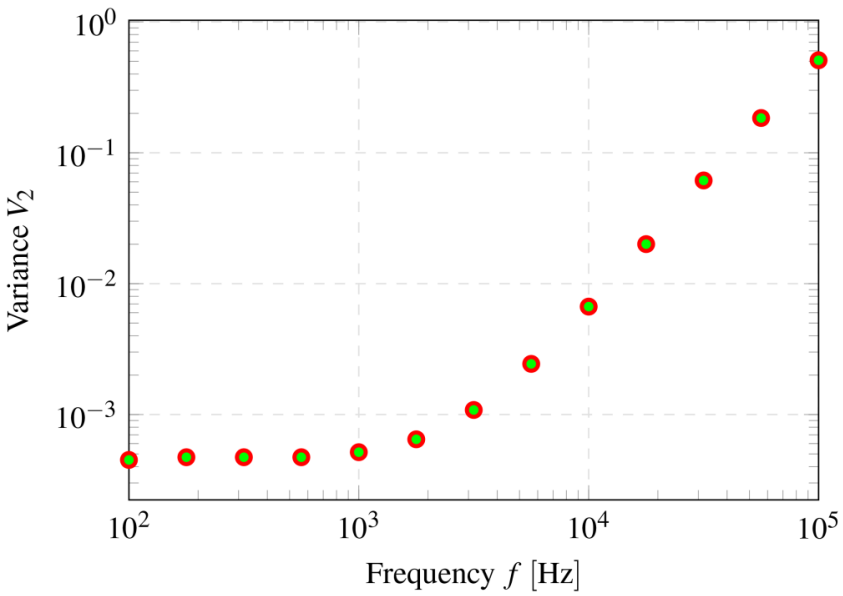

FIG. 5. Variance $V_{2}$ of the magnetic field in iron particles for different frequency values $f$ determined from a harmonic FEM on a unit cell with linear magnetic behavior for iron. Unit cell size: $L_{1}=50 \mu \mathrm{m}, L_{2}=49 \mu \mathrm{m}$.

Figure 5 shows the variance $V_{2}$, estimated from the FEM model, for different frequency values $f$ in the case of linear magnetic behavior for iron.

It can be concluded that, for low frequency values ( $f<10 \mathrm{kHz}$ ), the variance is less than $1 \%$, which means the uniformity assumption used in the homogenization models should provide accurate estimates for effective permeability and EC loss density. For higher frequencies $(f>10 \mathrm{kHz})$, the magnetic field induced by eddy currents (skin effect) cannot be neglected anymore, meaning that the developed models may become inaccurate.

In the case of nonlinear magnetic behavior for iron, the uniformity of the magnetic field remains valid.

\section{Effective magnetic behavior}

Figure 6 shows the effective permeability $\widetilde{\mu}$ of the unit cell for different inclusion sizes $L_{2}$ when iron exhibits a linear magnetic behavior.

It can be seen that the MG estimate $\widetilde{\mu}_{M G}$ [Eq. (2)] is very accurate in that case, which was expected since the magnetic field in the iron inclusion can be considered as uniform. The error between the homogenization model and the FEM (considered as the reference result) concerning the effective permeability is less than $0.5 \%$ (the higher the volume fraction $\xi_{2}$ is, the lower the error is).

For nonlinear magnetic behavior of iron, Fig. 7 shows the quasi-static magnetization response of the unit cell.

It can be concluded that the homogenization model is very accurate since the results are very close to the ones from FEM. The error in the predicted macroscopic induction $\overline{\mathbf{B}}$ is less than $0.5 \%$ in the linear part of the magnetization curve and even lower in the saturation part.

\section{EC loss density}

Figure 8 shows the predicted EC loss density $U$ of the unit cell when linear magnetic behavior of iron is considered, and for 


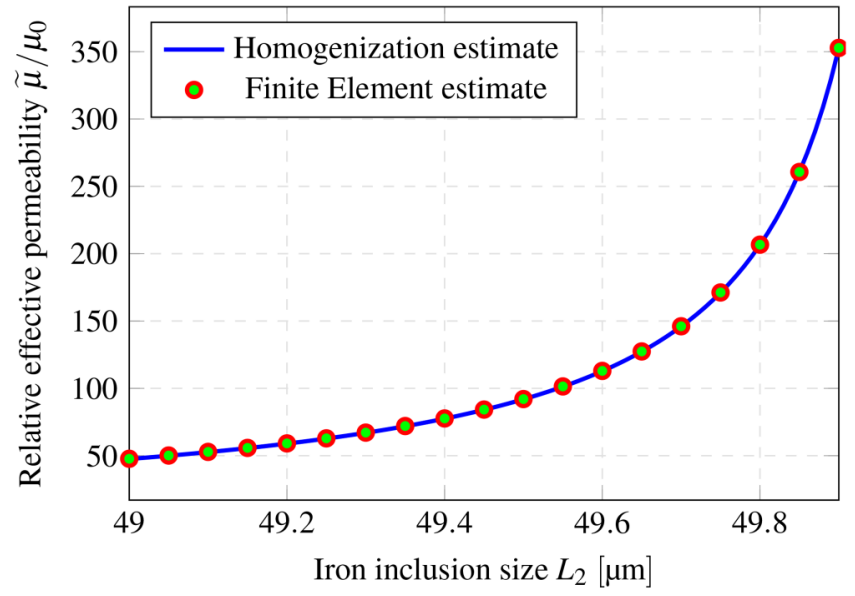

FIG. 6. Effective permeability with linear magnetic behavior of iron for different inclusion sizes $L_{2}$. Unit cell size: $L_{1}=50 \mu \mathrm{m}$.

different inclusion sizes $L_{2}$. The loading is a sinusoidal magnetic induction with a fixed magnitude of $1 T$ and a frequency of $1 \mathrm{kHz}$.

The homogenization estimate for EC loss density $U$ [Eq. (9)] gives very satisfying results. The error with FEM results is less than $0.1 \%$ and similarly to the observation for effective permeability, the higher the volume fraction $\xi_{2}$, the lower the error.

Figure 9 shows the EC loss density of the unit cell when nonlinear magnetic behavior for iron is considered, for different loadings. The loadings are sinusoidal macroscopic magnetic fields with different magnitudes $\bar{H}$ and a fixed frequency of $1 \mathrm{kHz}$.

Again, the homogenization model provides a very accurate estimate of EC loss density $U$ for the unit cell, even when the

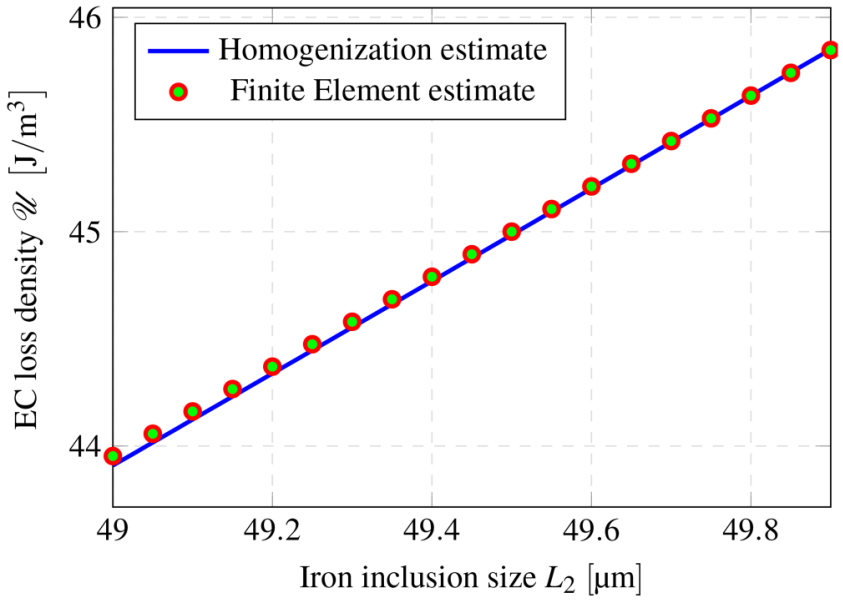

FIG. 8. EC loss density with linear magnetic behavior of iron for different iron inclusion sizes $L_{2}$. Unit cell size: $L_{1}=50 \mu \mathrm{m}$. Fixed magnitude of macroscopic flux density: $\bar{B}=1 \mathrm{~T}$. Frequency: $f=1 \mathrm{kHz}$

magnetic behavior of iron is nonlinear. However, it is noticed that homogenization systematically overestimates the EC loss density $U$ in the nonlinear case (Fig. 9), when compared to the reference value obtained by FEM. The error actually increases with the macroscopic magnetic field magnitude $\bar{H}$, being less than $0.1 \%$ for low magnitudes $(\bar{H}<15 \mathrm{kA} / \mathrm{m}$, which corresponds to the linear magnetic behavior for the iron particle) and increasing up to $1 \%$ for higher magnitudes $\bar{H}$ (when the iron particle attains magnetic saturation during the time period).

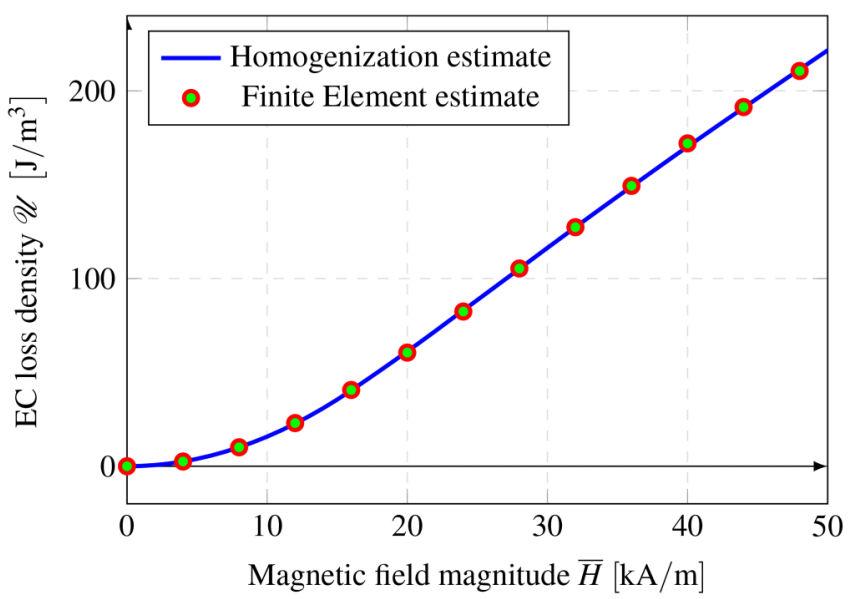

FIG. 9. EC loss density with nonlinear magnetic behavior of iron for different macroscopic magnetic field magnitudes $\bar{H}$. Unit cell size: $L_{1}=50 \mu \mathrm{m}$, $L_{1}=49 \mu \mathrm{m}$. Frequency: $f=1 \mathrm{kHz}$.
FIG. 7. Macroscopic magnetization response of the unit cell. Unit cell size: $L_{1}=50 \mu \mathrm{m}, L_{2}=49 \mu \mathrm{m}$. Frequency: $f=1 \mathrm{kHz}$. 


\section{Discussion}

These results show that the macroscopic behavior of a unit cell of SMC can be homogenized with analytical tools. The macroscopic magnetic behavior (effective permeability or magnetization curve) and EC loss density can be estimated with a very high accuracy.

The key assumption making this model accurate is the uniformity of the magnetic field in the particles. It means that the model remains accurate for high concentration SMC, for any size of particles (as long as the frequency does not bring skin effect), or for any (convex) magnetization curve for the particle. For more generic microstructures (particles of different sizes), a statistical approach of the size distribution ${ }^{34}$ showed interesting results in the linear case and may be transposed to handle nonlinear magnetic behavior too.

For higher frequencies with the appearance of skin effect, the use of numerical tools such as FEM would be necessary for determining the magnetic field and EC distribution.

In Sec. III C, a magnetic circuit made of SMC will be modeled. It will be verified that the homogenized properties are not only relevant for a unit cell of SMC but also for the study of a magnetic device.

\section{Magnetic circuit}

A magnetic circuit made of SMC such as the one depicted in Fig. 1 is modeled with FEM. Because of the symmetry, only half of the magnetic circuit is modeled.

Two Finite Element (FE) models are designed as:

- heterogeneous SMC: a FE model, taking into account every iron inclusions (with electrical conductivity) embedded in epoxy and

- homogeneous SMC: a FE model, using homogenized properties for SMC (no electrical conductivity).

If the magnetic circuit size was in the order of $\mathrm{cm}$ or $\mathrm{dm}$, the first FE model would be computationally massive because of the small inclusion size $(49 \mu \mathrm{m}$ in this study), especially for timedomain studies with nonlinear magnetic behavior. The magnetic circuit modeled in this paper has a size of $3 \mathrm{~mm}$ in order to handle a reasonably sized numerical system, but without loss of generality since the purpose of this section is to validate the homogenization models for the study of magnetic devices made of SMC.

For the second FE model using homogenized properties, it should be noted that the mesh required to model the geometry is much coarser, leading to a much smaller numerical system than for the first FE model. However, for the sake of comparing the two models, the geometry corresponding to every unit cell (a $50 \mu \mathrm{m}$ square) is still modeled and will be referred to as "virtual cell" later.

The same external sinusoidal current density (with frequency $f=1 \mathrm{kHz}$ ) is forced in the copper coils of both models.

\section{Linear magnetic behavior}

Since the magnetic behavior of iron is linear, the FEM studies are harmonic studies. For the homogeneous SMC model, the properties are modeled with a complex permeability $\widetilde{\mu}$,

$$
\widetilde{\mu}=\widetilde{\mu}_{M G}-\widetilde{\mu}_{E C}
$$

where $\widetilde{\mu}_{E C}$ models the EC losses. Following Ref. 24, the imaginary part of this complex permeability can be determined by loss equivalence,

$$
U=\pi \widetilde{\mu}_{E C} \overline{\mathbf{H}}^{*} \cdot \overline{\mathbf{H}}
$$

Then, using Eq. (8) leads to

$$
\widetilde{\mu}_{E C}=\frac{2 \pi}{3} f \xi_{2} L_{2}^{2} \sigma_{2}\left(\frac{\mu_{1} \mu_{2}}{\left(\mu_{2}+\mu_{1}\right)-\left(\mu_{2}-\mu_{1}\right) \xi_{2}}\right)^{2} .
$$

In order to quantitatively compare the prediction of EC losses from the homogenized SMC, Eq. (17) will be averaged on every virtual cell with

$$
\widetilde{U}=\pi \widetilde{\mu}_{E C}\left\langle\underline{\mathbf{H}^{*}}(\mathbf{x}) \cdot \underline{\mathbf{H}}(\mathbf{x})\right\rangle_{v c}
$$

where the operator $\langle\cdot\rangle_{v c}$ indicates a volume average over a virtual cell.

Figure 10 shows the distribution of magnetic induction for both models. It can be seen that the homogenized model predicts a magnetic induction distribution very similar to the heterogeneous

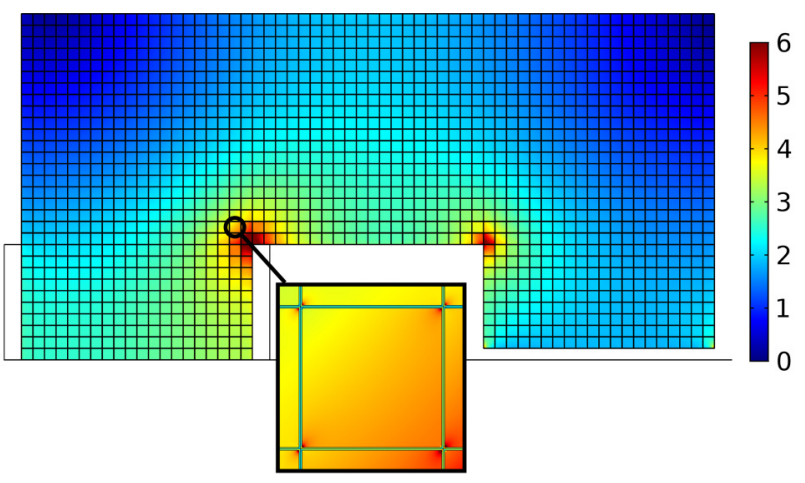

(a)

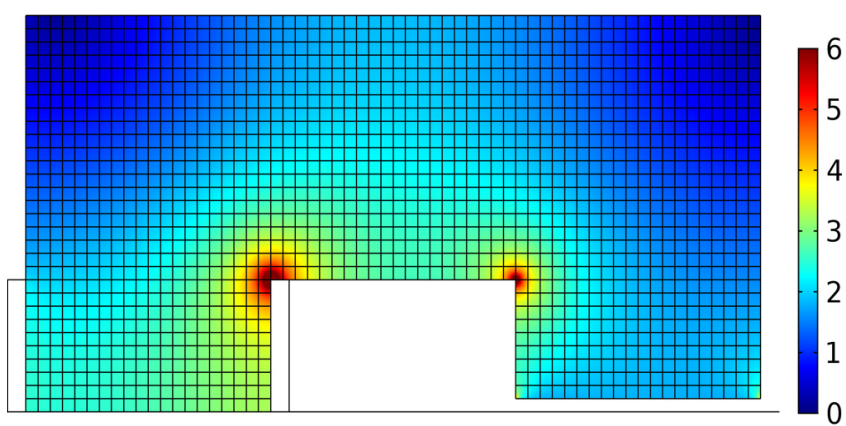

(b)

FIG. 10. Norm of the harmonic magnetic induction $\|\mathbf{B}\|$ (unit: $\mathbf{T}$ ) in the magnetic circuit with linear magnetic properties. (a) Heterogeneous SMC (maximum induction: 16.0 T). (b) Homogeneous SMC (maximum induction: 14.6 T). Colormaps are saturated (data with $\|\mathbf{B}\|>6 T$ exhibit the same color as $\|\mathbf{B}\|=6 T)$. 
model, demonstrating that the homogenized permeability accurately represents the macroscopic magnetic behavior in that case.

By looking to the heterogeneous model in Fig. 10(a), it seems that the assumption of a uniform magnetic field in iron inclusions is valid for most of the inclusions. Only a few of them, mainly located in the inner corners of the magnetic circuit, show a heterogeneous magnetic distribution. It is expected that the homogenized model will be less accurate for these inclusions because the homogenization assumption fails.

Distribution of magnetic induction in the airgap (red line in Fig. 1) is also verified in Fig. 11. It can be observed that both the real and imaginary parts of the magnetic induction in the airgap are correctly predicted by the homogenized model, the error staying below $1 \%$. It definitely confirms that homogenizing the magnetic circuit with a complex permeability is a valid approach in this study.

The distribution of the out-of-plane ( $z$ direction) eddy current in the heterogeneous model (with no current in epoxy) is shown in Fig. 12. Each inclusion exhibits a null net current, showing a perfect insulation condition. The inclusions exhibiting the highest induced current density are located in the inner corners of the magnetic circuit, due to the high magnetic induction value as shown in Fig. 10(a).

Figure 13(a) shows the EC loss density $U$ determined for every unit cell from Eq. (6) on the heterogeneous model and Fig. 13(b) shows the EC loss density $\widetilde{U}$ predicted from Eq. (19) on the homogeneous model. Again, the EC loss distribution in the magnetic circuit seems to be accurately predicted by the homogenized model.

For the sake of evaluating the accuracy of the homogenization model, the relative error $\xi$ (in \%) of EC loss density predicted by
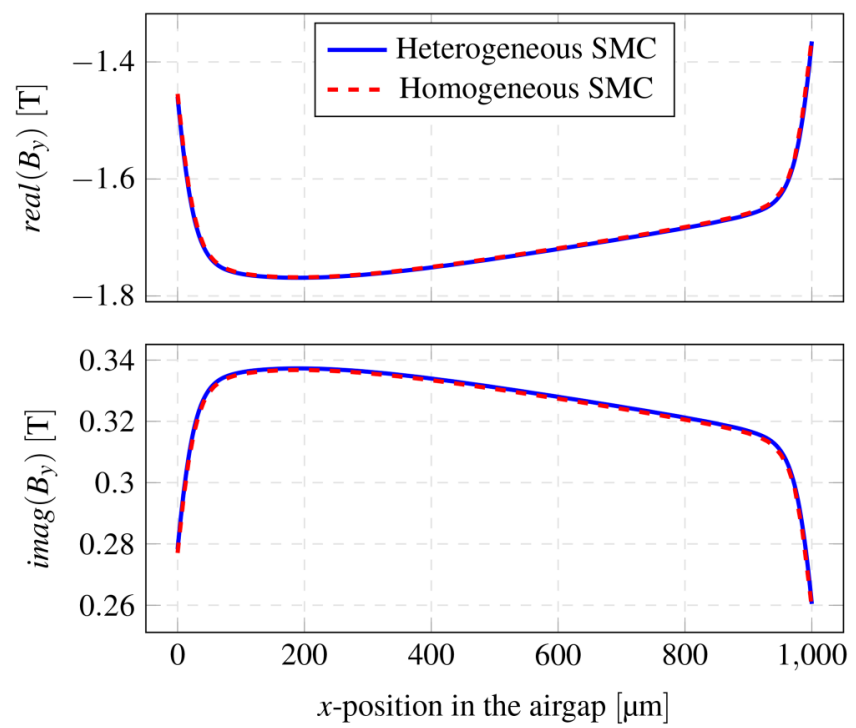

FIG. 11. Real part (top) and imaginary part (bottom) of the magnetic induction $B_{y}$ in the airgap as a function of the $x$-position. Solid lines: heterogeneous SMC. Dashed lines: homogeneous SMC.

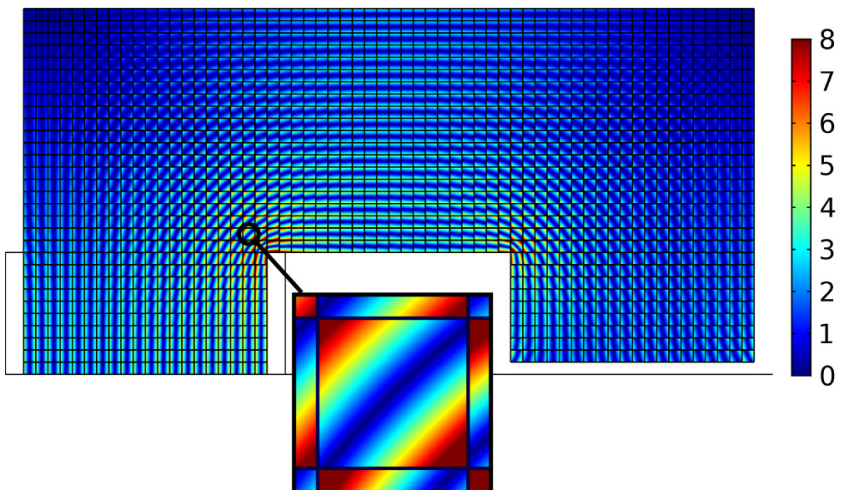

FIG. 12. Heterogeneous SMC: modulus of the harmonic induced current density $J_{z}$ (unit: $A / \mathrm{mm}^{2}$ ) in the magnetic circuit. Colormap is saturated (data with $J_{z}>8 \mathrm{~A} / \mathrm{mm}^{2}$ exhibit the same color as $J_{z}=8 \mathrm{~A} / \mathrm{mm}^{2}$ ) (maximum current density: $18.2 \mathrm{~A} / \mathrm{mm}^{2}$ ).

the homogenized model $(\widetilde{U})$ compared to the one from heterogeneous model $(U)$ is shown in Fig. 14,

$$
\xi(\%)=\frac{\widetilde{U}-U}{U} \cdot 100 .
$$

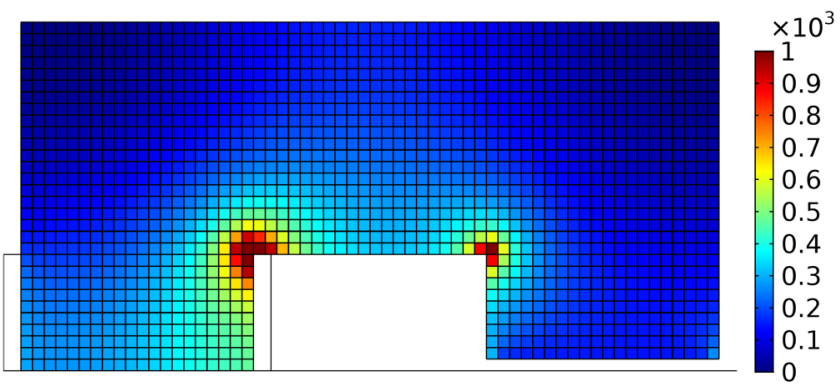

(a)

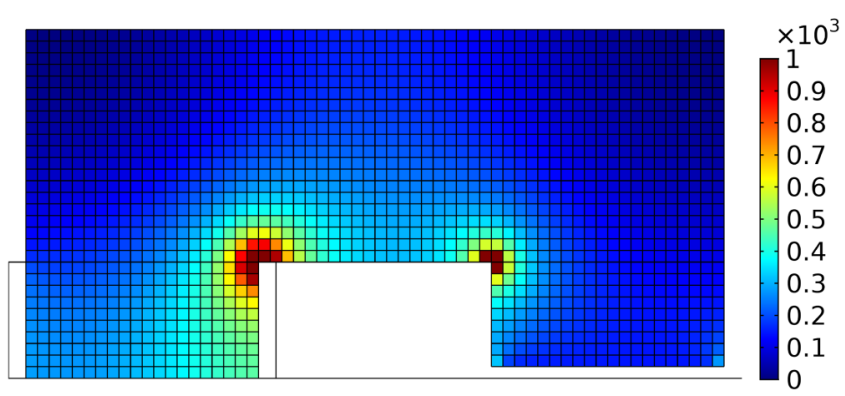

(b)

FIG. 13. EC loss density $U$ (averaged for every cell or virtual cell, unit: $\mathrm{J} / \mathrm{m}^{3}$ ). (a) Heterogeneous SMC (maximum value: $1850 \mathrm{~J} / \mathrm{m}^{3}$ ). (b) Homogeneous SMC (maximum value: $1690 \mathrm{~J} / \mathrm{m}^{3}$ ). Colormaps are saturated (data with $U>$ $1000 \mathrm{~J} / \mathrm{m}^{3}$ exhibit the same color as $U=1000 \mathrm{~J} / \mathrm{m}^{3}$ ). 


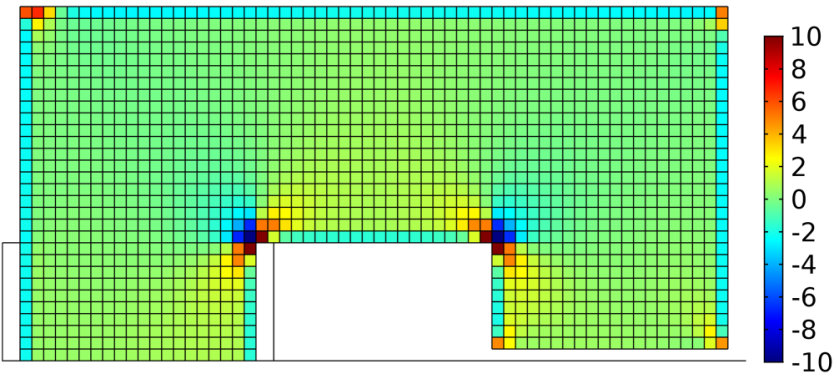

FIG. 14. Relative error $\xi$ (unit: \%) on EC loss density estimation $\widetilde{U}$. Colormap is saturated (data with Error $(\%)>10 \%$ exhibit the same color as Error $(\%)=$ $10 \%$ and data with Error $(\%)<-10 \%$ exhibit the same color as Error $(\%)=-10 \%)$ (extremum values: $-11 \%$ and $19 \%$ ).

It should be noted that only 27 virtual cells (which means $1.7 \%$ of the total number of virtual cells which is 1580) exhibit an absolute error on the EC loss density prediction of more than $3 \%$. These cells are essentially located in the inner corners of the magnetic circuit geometry, where the magnetic field varies spatially rapidly (see Fig. 10) and which makes the homogenization assumption of a unit cell being a good representative volume element (RVE) fail.

Finally, the total EC loss (linear density in the out-of-plane direction) on the whole magnetic circuit is evaluated. The heterogeneous model gives a value of $0.72215 \mathrm{~J} / \mathrm{mm}$ while the homogeneous model predicts a value of $0.72491 \mathrm{~J} / \mathrm{mm}$. The error on total EC loss prediction for the magnetic circuit is less than $0.5 \%$.

These results show that the developed homogenization model is very accurate for modeling SMC in the case of linear magnetic behavior, both from the point of view of magnetic permeability and for the evaluation of EC losses. Section III C 2 will also verify the accuracy of the model when nonlinear magnetization of iron is considered.

\section{Nonlinear magnetic behavior}

Since the magnetic behavior of iron is nonlinear, the FEM studies are time-dependent studies. Transient behavior is discarded and only steady-state results are shown in this section. An external sinusoidal current density is forced in the coil,

$$
J_{z}^{e x t}(t)= \pm J_{0} \cdot \sin (2 \pi f t)
$$

$J_{0}$ value is chosen so that a major part of the magnetic circuit reaches magnetic saturation in order to differentiate from the previous study (linear magnetic behavior).

The homogenized model is a lossless model, with a posteriori estimation of EC losses. In order to quantitatively compare the prediction of EC losses from the homogenized SMC, Eq. (14) will be averaged on every virtual cell with

$$
\widetilde{U}=\frac{\xi_{2} \sigma_{2} L_{2}^{2} \alpha^{2}}{12} \int_{0}^{T}\left\|\frac{\partial\langle\mathbf{B}(t)\rangle_{\nu c}}{\partial t}\right\|^{2} d t,
$$

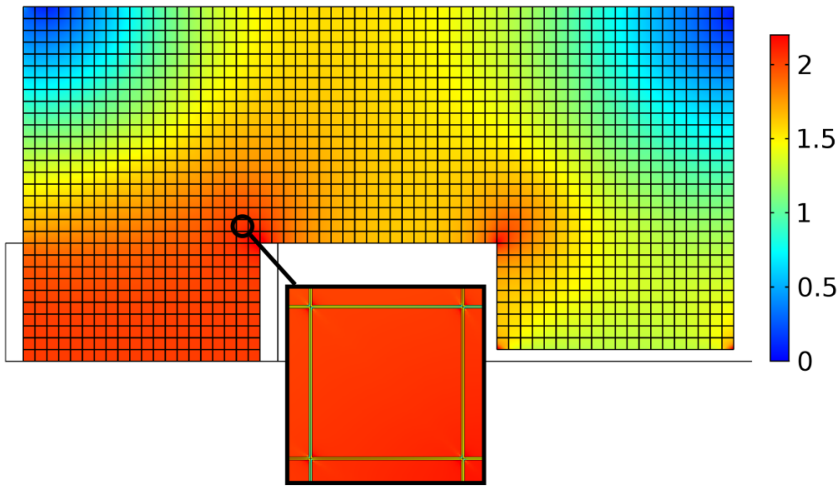

(a)

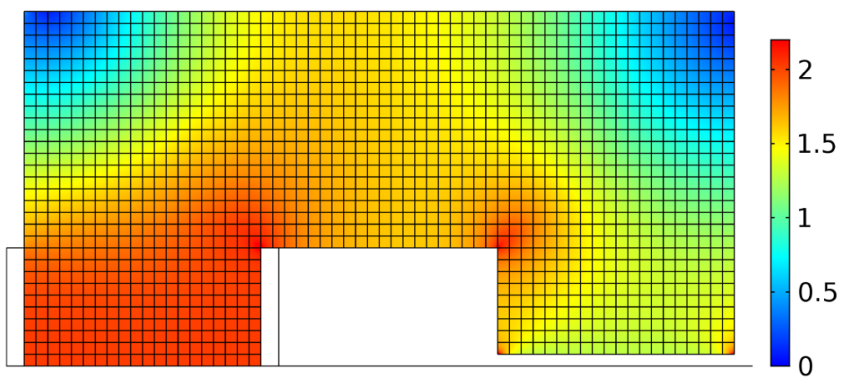

(b)

FIG. 15. Norm of the magnetic induction $\|\mathbf{B}\|$ (unit: $\mathrm{T}$ ) in the magnetic circuit at $t=0.25 \mathrm{~ms}$ (steady-state). (a) Heterogeneous SMC (maximum induction: $3.4 \mathrm{~T}$ ). (b) Homogeneous SMC (maximum induction: $3.0 \mathrm{~T}$ ). Colormaps are saturated (data with $\|\mathbf{B}\|>2.2 T$ exhibit the same color as $\|\mathbf{B}\|=2.2 \mathrm{~T}$ ).

where the operator $\langle\cdot\rangle_{v c}$ indicates a volume average over a virtual cell.

Figures 15(a) and 15(b) show the distribution of magnetic induction for both models, at the same instant (when the external sinusoidal current density in the coil reaches its maximum). It can be seen that the homogenized model predicts a magnetic field distribution very similar to the one from the heterogeneous model, demonstrating that the homogenized magnetization curve (shown in Fig. 7) accurately represents the macroscopic magnetic behavior in that case.

The time evolution of magnetic induction at the center of the airgap is also verified in Fig. 16. It can be seen that homogenizing the magnetic circuit is a valid approach even with nonlinear magnetic behavior, the error staying below $2 \%$.

However, it is observed that the homogenized model slightly overestimates the magnitude of the magnetic induction (for the same current applied in the coil) by $2 \%$ on average. This can be explained by the fact that the SMC in the homogenized model does not exhibit any loss (null macroscopic conductivity), while the heterogeneous model exhibits some EC loss in every cell.

Figure 17 shows the distribution of the out-of-plane ( $z$ direction) eddy current in the heterogeneous model at $t=0.5 \mathrm{~ms}$ (steady-state). 


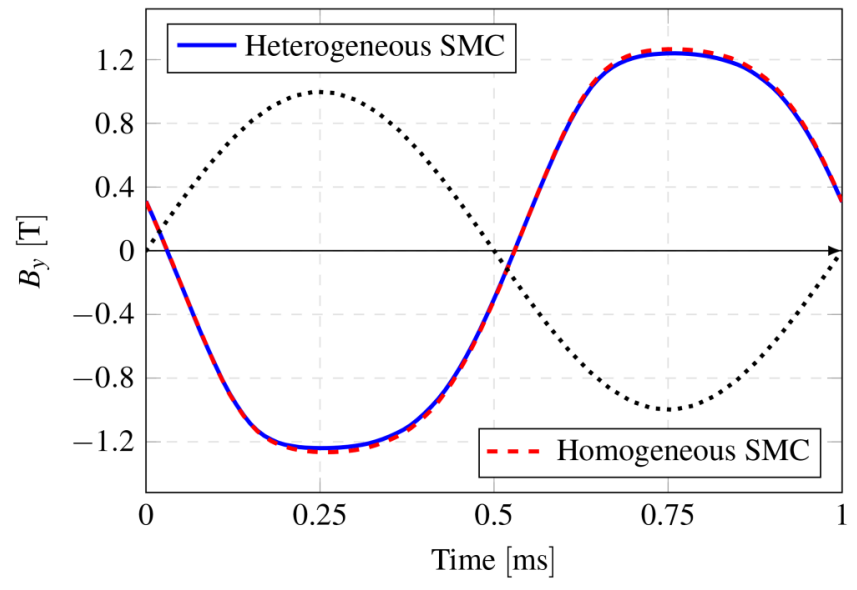

FIG. 16. Time evolution of the magnetic induction $B_{y}$ in the center of the airgap during one period (steady-state). Solid line: heterogeneous SMC. Dashed line: homogeneous SMC. Dotted line: external current density in the coil (given as a reference for visualizing the phase shifting).

Each inclusion exhibits a null net current at any time, showing a perfect insulation condition.

Figure 18(a) shows the EC loss density $U$ determined for every unit cell from Eq. (5) with the heterogeneous model and Fig. 18(b) shows the EC loss density $\widetilde{U}$ predicted from Eq. (22) using the homogeneous model. Similarly to the conclusions with linear magnetic behavior, the EC loss density is accurately predicted by the homogenized model with nonlinear magnetic behavior.

Figure 19 shows the error between the two models [see Eq. (20)]. It should be noted that only 56 virtual cells (which means $3.5 \%$ of the total number of virtual cells) exhibit an absolute error on the EC loss density prediction of more than $6 \%$.

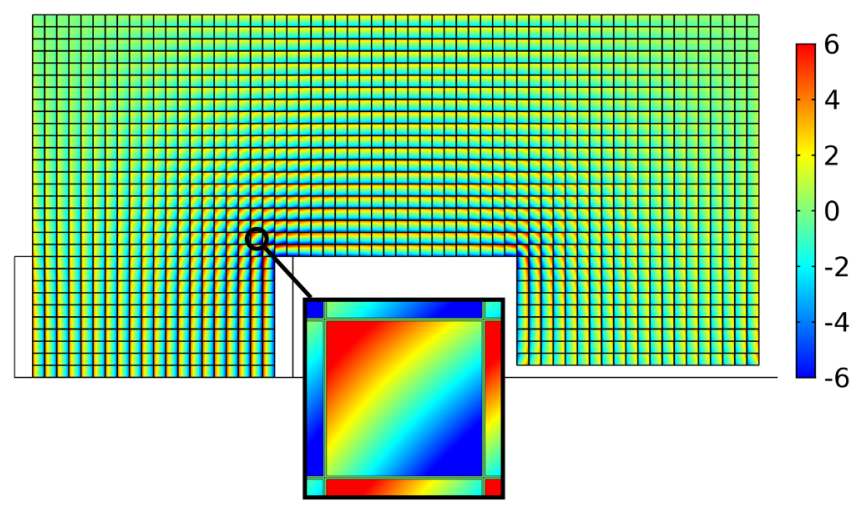

FIG. 17. Heterogeneous SMC: induced current density $J_{z}$ (unit: $A / \mathrm{mm}^{2}$ ) in the magnetic circuit at $t=0.5 \mathrm{~ms}$ (steady-state). Colormap is saturated (data with $J_{z}>6 \mathrm{~A} / \mathrm{mm}^{2}$ exhibit the same color as $J_{z}=6 \mathrm{~A} / \mathrm{mm}^{2}$ ) (maximum current density: $13.6 \mathrm{~A} / \mathrm{mm}^{2}$ ).

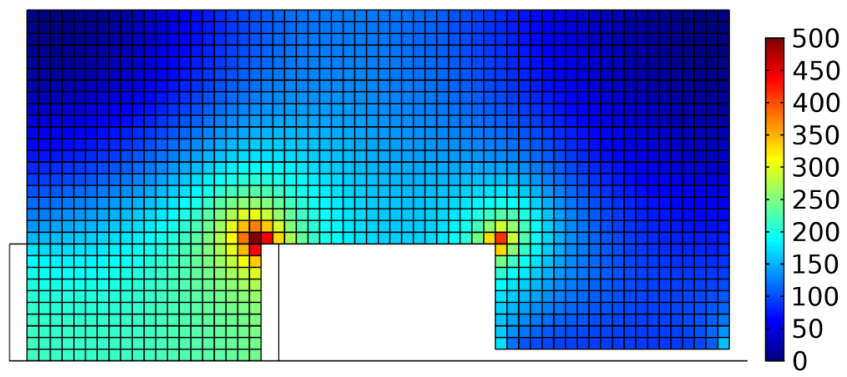

(a)

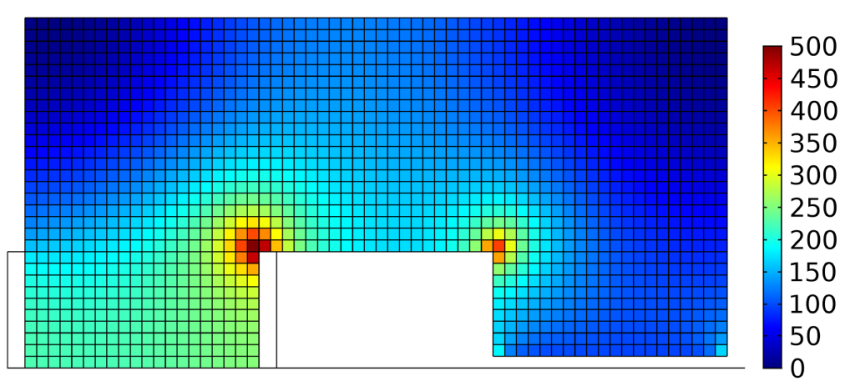

(b)

FIG. 18. EC loss density $U$ (averaged for every cell or virtual cell, unit: $\mathrm{J} / \mathrm{m}^{3}$ ). (a) Heterogeneous SMC (maximum value: $538 \mathrm{~J} / \mathrm{m}^{3}$ ). (b) Homogeneous SMC (maximum value: $527 \mathrm{~J} / \mathrm{m}^{3}$ ). Colormaps are saturated (data with $U>$ $500 \mathrm{~J} / \mathrm{m}^{3}$ exhibit the same color as $U=500 \mathrm{~J} / \mathrm{m}^{3}$ ).

Finally, the total EC loss (linear density in the out-of-plane direction) on the whole magnetic circuit is evaluated. The heterogeneous model gives a value of $0.48158 \mathrm{~J} / \mathrm{mm}$ while the homogeneous model predicts a value of $0.50356 \mathrm{~J} / \mathrm{mm}$. The overestimation of total EC loss prediction for the magnetic circuit is close to $4.5 \%$. It is observed that EC loss density $U$ is overestimated on most areas of the magnetic circuit, and the error is globally much higher than the one observed in the linear magnetic case. A similar observation was made on the magnetic induction distribution in Fig. 15.

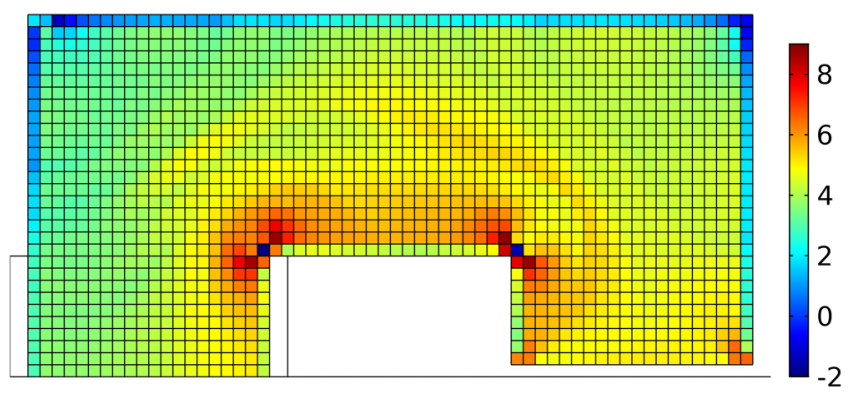

FIG. 19. Relative error (unit: \%) on EC loss density estimation $\widetilde{U}$ with the homogeneous model compared to the value from the heterogeneous model. 
An error of $2 \%$ on the magnetic induction will actually induce a $4 \%$ error on the EC losses because of their quadratic dependence on $\overline{\mathbf{B}}$ [see Eq. (14)].

But overall, these results confirm that the developed homogenization model is able to predict the EC losses in SMC with a satisfying accuracy even in the case of nonlinear magnetic behavior.

\section{CONCLUSIONS}

Magnetic properties of high concentration SMC can be estimated with the presented semi-analytical homogenization models. Both magnetic behavior and the level of EC losses are accurately estimated, even with nonlinear magnetization for iron particles. These models can deal with a nonuniform magnetic field (the field is in-plane in this study) in the composite, which means they can also be used for the study of 3D SMC.

Compared to the usual numerical approaches used when nonlinear magnetic behavior is considered, the results show that the semi-analytical approach provides an accurate estimate of EC losses.

Full-field FEM computations have been performed on a tiny $2 \mathrm{D}$ magnetic circuit in order to validate the homogenization approach. Results show that the homogenized behavior of SMC can be implemented in FEM computations in order to greatly reduce the size of the problem (and thus greatly reduce the computation time).

The models keep their accuracy as long as skin effect can be neglected (in the presented application, the models are very accurate when the frequency is less than $20 \mathrm{kHz}$ ). For higher frequencies with nonlinear magnetic behavior, numerical models are required since an analytical description of EC losses is not possible anymore.

A possible improvement of the models in the future would be to consider more realistic microstructures of SMC bringing some randomness in the inclusion sizes and shapes. It is anticipated that the models will still remain accurate because the key assumption of the models (a uniform magnetic field in the inclusions) should still be verified. The EC losses will be more difficult to homogenize with random inclusions, but strategies that can consider randomness in microstructures have shown interesting potential. ${ }^{34}$

\section{ACKNOWLEDGMENTS}

This work was partially supported by CAPES/COFECUB under Project No. 88881.191763/2018-01.

\section{DATA AVAILABILITY}

The data that support the findings of this study are available from the corresponding author upon reasonable request.

\section{REFERENCES}

${ }^{1}$ L. M. Bollig, P. J. Hilpisch, G. S. Mowry, and B. B. Nelson-Cheeseman, J. Magn. Magn. Mater. 442, 97-101 (2017).

${ }^{2}$ Y. Ito, H. Igarashi, M. Suzuki, Y. Iwasaki, and K. Kawano, IEEE Trans. Magn. 52, 9400804 (2016).

${ }^{3}$ A. Jack, in IEE Colloquium on New Magnetic Materials-Bonded Iron, Lamination Steels, Sintered Iron and Permanent Magnets (IET, 1998).

${ }^{4}$ C. Liu, J. Zhu, Y. Wang, G. Lei, and Y. Guo, IEEE Trans. Appl. Supercond. 26, 5203505 (2016).

${ }^{\mathbf{5}}$ G. Bertotti, IEEE Trans. Magn. 24, 621-630 (1988).

${ }^{6} \mathrm{G}$. Bertotti, Hysteresis in Magnetism: For Physicists, Materials Scientists, and Engineers (Academic Press, 2008).

${ }^{7}$ M. A. Willard, T. Francavilla, and V. G. Harris, J. Appl. Phys. 97, 10 F502 (2005).

${ }^{\mathbf{8}}$ O. de la Barrière, C. Appino, F. Fiorillo, C. Ragusa, H. Ben Ahmed, M. Gabsi, F. Mazaleyrat, and M. Lobue, J. Appl. Phys. 109, 17A331 (2011).

${ }^{9}$ J. Vesa and P. Rasilo, IEEE Trans. Magn. 56, 1 (2020).

${ }^{10}$ Y. Gao, T. Fujiki, H. Dozono, K. Muramatsu, W. Guan, J. Yuan, C. Tian, and B. Chen, IEEE Trans. Magn. 54, 1-4 (2018).

${ }^{11}$ A. Maruo and H. Igarashi, IEEE Trans. Magn. 55, 2002205 (2019).

${ }^{12}$ Y. Sato, T. Shimotani, and H. Igarashi, IEEE Trans. Magn. 53, 1-4 (2017).

${ }^{13}$ O. Bottauscio and A. Manzin, J. Comput. Phys. 253, 1-17 (2013).

${ }^{14} \mathrm{R}$. Pal, Electromagnetic, Mechanical, and Transport Properties of Composite Materials (CRC Press, 2015).

${ }^{15}$ P. Queffelec, D. Bariou, and P. Gelin, IEEE Trans. Magn. 41, 17-23 (2005).

${ }^{16} \mathrm{C}$. Brosseau and A. Beroual, Prog. Mater. Sci. 48, 373-456 (2003).

${ }^{17}$ V. Preault, R. Corcolle, L. Daniel, and L. Pichon, IEEE Trans. Electromagn. Compat. 55, 1178-1186 (2013).

${ }^{18} \mathrm{~K}$. Abeywickrama, T. Daszczynski, Y. Serdyuk, and S. Gubanski, IEEE Trans. Magn. 44, 438-444 (2008).

${ }^{19}$ H. Hamzehbahmani, P. Anderson, J. Hall, and D. Fox, IEEE Trans. Power Delivery 29, 642-650 (2014).

${ }^{20}$ O. Moreau, L. Popiel, and J. Pages, IEEE Trans. Magn. 34, 3616-3619 (1998).

${ }^{21} \mathrm{X}$. Nan and C. R. Sullivan, in 2005 IEEE 36th Power Electronics Specialists Conference (IEEE, 2005), pp. 613-618.

${ }^{22}$ A. Baghel, B. Sai Ram, L. Daniel, S. Kulkarni, G. Krebs, J. Blumenfeld, and L. Santandrea, Comput. Mater. Sci. 166, 96 (2019).

${ }^{23}$ H. Igarashi, IEEE Trans. Magn. 53, 1 (2017).

${ }^{24}$ X. Ren, R. Corcolle, and L. Daniel, IEEE Trans. Magn. 52, 6301609 (2016).

${ }^{25}$ Y. Ito and H. Igarashi, IEEE Trans. Magn. 49, 1953 (2013).

${ }^{26}$ S. K. Mukerji, M. George, M. B. Ramamurthy, and K. Asaduzzaman, Prog. Electromagn. Res. B 7, 117 (2008).

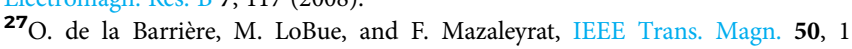
(2014).

${ }^{28}$ X. Ren, R. Corcolle, and L. Daniel, Eur. Phys. J. Appl. Phys. 73, 20902 (2016).

${ }^{29}$ K. Matouš, M. G. Geers, V. G. Kouznetsova, and A. Gillman, J. Comput. Phys. 330, 192 (2017).

30J. C. Maxwell Garnett, Philos. Trans. R. Soc. London Ser. A 73, 443-445 (1904).

${ }^{31}$ COMSOL Inc, AC/DC module, version 5.4.

${ }^{32}$ R. Corcolle, L. Daniel, and F. Bouillault, J. Appl. Phys. 105, 123913 (2009).

${ }^{33}$ R. Corcolle, L. Daniel, and F. Bouillault, IEEE Trans. Magn. 44, 17 (2008).

${ }^{34}$ X. Ren, R. Corcolle, and L. Daniel, J. Appl. Phys. 123, 235109 (2018). 